\title{
THE TOPOLOGY OF CERTAIN SPACES OF PATHS ON A COMPACT SYMMETRIC $\operatorname{SPACE}\left({ }^{1}\right)$ \\ BY \\ LAWRENCE CONLON(2)
}

Introduction. In this paper we apply the theory of Bott and Samelson [6] to the study of the integral singular homology of certain spaces of paths on a compact symmetric space. It should be noted that a special case of one of our theorems (Theorem 2.1) has been obtained independently by S. Araki and will appear in his forthcoming study of the Bott-Samelson $K$-cycles associated to symmetric spaces [2]. Some of the technical lemmas involved in the proof of Theorem 2.2 are also known to Araki, but are used by him for quite different purposes.

The spaces of paths to be considered are those associated to the variationally complete group actions described by Hermann in [7]. Thus if $G$ is a compact connected Lie group and $K, H$ are symmetric subgroups (for our purposes a symmetric subgroup is the full fixed point group of an involutive automorphism of $G$ ) then we let $N$ be a $K$-orbit in $G / H$ and set $\Omega=\Omega(G / H ; x, N)$, the space of paths in $G / H$ which start at the point $x$ and terminate on $N$. Our principal results will be a complete determination of the $K$-cycles in $\Omega$ (cf. [6, pp. 969-972])

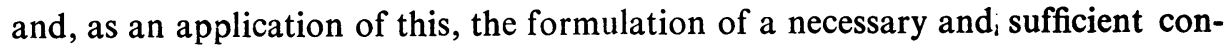
dition that the singular homology $H_{*}(\Omega)$ be free of torsion.

Any effective application of the Bott-Samelson theory to $\Omega$ will require a description of the distribution and defects of the $K$-orbits in $G / H$. For the case $K=H$ it is known (cf. [6]) that such a description is provided by a maximal torus of $G / K$ together with a certain "diagram" of singular subtori determined by the root system of $G / K$. We generalize this situation to the "symmetric triad" $(G ; K, H)$ by selecting a geodesically imbedded torus $T$ in $G / H$ which meets all the $K$-orbits and meets them orthogonally (cf. [8]), the singular points of which again fall into a finite union of subtori. Some propositions of Siebenthal proven in [12] then provide the key to a complete description of these singular

Received by the editors April 1, 1963.

(1) The results in this paper are from my doctoral dissertation, submitted to Harvard University in January, 1963. I am especially grateful to Professor Raoul Bott who directed this research and whose advice was very helpful in the preparation of this paper. I am also grateful to the referee for many helpful suggestions.

(2) During this research the author held a National Science Foundation Graduate Fellowship. 
subtori. More precisely, these propositions enable us to define certain affine functionals on the universal covering $t$ of $T$. These functionals (called the roots of the triad $(G ; K, H))$ determine a diagram of singular hyperplanes $p$ of $t$, each having a positive multiplicity $m(p)$, and this diagram plays a role in the general context entirely analogous to that played by the usual root diagram in the case $K=H$. The precise theorem is:

TheOREM 1.1. Let $Y \in t$ and $y=\exp (Y) \in T$. Then the defect $d(y)$ (i.e., the non-negative difference between the dimension of the K-orbit of $y$ and the maximal dimension of any K-orbit) is the number of singular planes, counted with multiplicity, which contain $Y$.

This material is developed in $\S \mathrm{I}$.

These root diagrams enable us to prove a complete structure theorem for the Bott-Samelson $K$-cycles in $\Omega$. If $P=\left(p_{1}, \cdots, p_{r}\right)$ is a finite sequence of singular planes from the diagram in $t$, then the $K$-cycle

$$
\Gamma_{P}=K_{1} \times_{K_{T}} \cdots \times_{K_{T}}\left(K_{r} / K_{T}\right)
$$

is defined by taking $K_{i}$ as the subgroup of $K$ which leaves $\exp \left(p_{i}\right)$ pointwise fixed and $K_{T}$ the subgroup of $K$ which leaves $T$ pointwise fixed. Set $P_{i}=\left(p_{1}, \cdots, p_{i}\right)$, $i<r$, and $\Gamma_{i}=\Gamma_{P_{i}}$. The structure theorem for $\Gamma_{P}$ can now be stated.

THEOREM 2.1. There is a sequence of locally trivial fibrations

$$
\Gamma \underset{\pi_{r}}{\longrightarrow} \Gamma_{r-1} \underset{\pi_{r-1}}{\longrightarrow} \cdots \underset{\pi_{2}}{\longrightarrow} \Gamma_{1}=S^{m\left(p_{1}\right)}
$$

with the fiber of $\pi_{i}$ homeomorphic to the sphere $S^{m\left(p_{i}\right)}$. Each fibration $\pi_{i}$ admits a canonical global cross-section $s_{i}$. The sphere-bundle

$$
\Gamma_{i} \underset{\pi_{i}}{\longrightarrow} \Gamma_{i-1}
$$

is isomorphic to the associated unit sphere bundle of a vector bundle $E_{i}+1$ (where 1 denotes the trivial line bundle) over $\Gamma_{i-1}$. Here we have

$$
E_{i}=K_{1} \times{ }_{K_{T}} \cdots \times_{K_{T}} K_{i-1} \times{ }_{K_{T}} V_{i}
$$

where $V_{i}$ is the orthogonal complement of $\mathfrak{f}_{T}$ in $\mathfrak{\mathfrak { l }}_{i}$ (the respective Lie algebras of $K_{T}$ and $K_{i}$ ) and the left action of $K_{T}$ on $V_{i}$ is the adjoint action.

This is the theorem which Araki [2] also obtains, though he considers only the important case $K=H$.

Theorem 2.1 is proven in $\S \mathrm{II}$. The remainder of that section is devoted to a study of the topology of $\Gamma_{P}$. We give particular attention to the orientability of $\Gamma_{P}$, approaching this question by a study of the Whitney classes $w_{1}\left(E_{i}\right)$. We are led to define a "regularity" condition on $(G ; K, H)$ by requiring certain relations among the roots of the triad. The exact formulation of this condition must be 
postponed until these root systems have been discussed in greater detail, but the theorem which motivates the definition can be given here.

THEOREM 2.2. If $(G ; K, H)$ is regular, then every $\Gamma_{P}$ is homologically torsionfree (and so, in particular, is orientable). Conversely, if the regularity condition is not fulfilled, then some $\Gamma_{P}$ is nonorientable.

In §III we apply these considerations to a study of the torsion in $H_{*}(\Omega)$. It is well known from the Morse theory that if each transversal geodesic segment in $\Omega$ has even Morse index, then $H_{*}(\Omega)$ has only even dimensional classes and no torsion. By the variational completeness of the action of $K$ on $G / H$ together with Theorem 1.1, this condition is equivalent to demanding that all singular planes in $\mathfrak{t}$ have even multiplicity. One might conjecture that whenever this condition fails, torsion will occur in $H_{*}(\Omega)$, but this turns out to be false. Theorem 2.2 together with Theorem I of [6] assures us that whenever $(G ; K, H)$ is regular the group $H_{*}(\Omega)$ will be free-abelian, and there are a number of examples of regular triads whose root diagrams contain planes of odd multiplicity, even of multiplicity one. On the other hand, if the triad fails to be regular, then an argument using the $K$-cycles will exhibit the presence of nonzero two-torsion in $H_{*}(\Omega)$. This will complete the proof of the following theorem:

THEOREM 3.1. $H_{*}(\Omega)$ is torsion-free if and only if $(G ; K, H)$ is regular.

$\S$ III will be concluded by a brief discussion of the case $K=H$.

In all that follows we suppose a choice once and for all of a left and right invariant Riemannian metric on $G$. This yields an invariant inner product $\langle$, in the Lie algebra $g$ of $G$. If $U$ is a Lie subgroup of $G$ we denote by $U_{0}$ the identity component of $U$.

I. The roots of $(G ; K, H)$.

1. A special case. Before we can define the root system of a triad it is necessary to investigate a certain special class of symmetric triads. Let $A$ be any automorphism of $G$ and consider in $G \times G$ the following symmetric subgroups:

$$
\begin{aligned}
D & =\{(x, x): x \in G\}, \\
D_{A} & =\{(x, A(x)): x \in G\} .
\end{aligned}
$$

These are the respective fixed point groups of the involutions

$$
\begin{aligned}
& (x, y) \rightarrow(y, x), \\
& (x, y) \rightarrow\left(A^{-1}(y), A(x)\right) .
\end{aligned}
$$

Projection onto the first coordinate defines an isomorphism of $D_{A}$ with $G$ and the map of $G \times G$ to $G$ defined by $(x, y) \rightarrow y x^{-1}$ defines a diffeomorphism of $(G \times G) / D$ 
with $G$. Under these identifications the action of $D_{A}$ on $(G \times G) / D$ becomes the action of $G$ on itself defined by

$$
x \cdot y=A(x) y x^{-1}, \quad \text { for all } x, y \in G .
$$

We call this the $A$-twisted action of $G$ on itself.

Let $\mathfrak{f}_{A}$ be the subalgebra of $g$ left pointwise fixed by $A$ and let $\mathrm{t}_{A}$ be a maximal abelian subalgebra of $\mathfrak{f}_{A}$. It is not hard to show (and will follow from Proposition 1.4) that the orbits of the above action all meet the torus $T_{A}=\exp \left(\mathrm{t}_{A}\right)$ and meet it orthogonally. Thus to carry out the program sketched in the introduction for the special case of $A$-twisted actions we must describe the singular subtori of $T_{A}$. For this we will use a suitable family of affine functionals on $t_{A}$. These have been defined by Siebenthal [10] although for a different purpose and from a somewhat different point of view.

We will give a restatement of Siebenthal's results (the reference is [10], Chapter II, §3, and Chapter III, §1) suitably modified to fit our context. To carry out the modifications for himself the reader need only note that for a suitable finite extension $H$ of $G$ the automorphism $A$ of $G$ becomes the action $\operatorname{Ad}(x)$ on the identity component $H_{0}=G$ for a suitable $x \in H$. Siebenthal's propositions are:

Proposition S-1(p.56). $T_{A}$ is contained in a unique maximal torus $T$ of $G$. Hence $T$ is invariant under $A$.

Proposition S-2 (p. 62). The roots of $G$ relative to $T$ fall into equivalence classes consisting of those roots whose restrictions to $t_{A}$ are equal. $A^{-1}$ permutes the elements of such a class cyclically.

Each of these equivalence classes will be called a cycle. If $e_{1}, \ldots, e_{k}$ are the 2-planes in $m=t^{\perp}$ ( $t$ denotes the Lie algebra of $T$ in $g$ ) corresponding to the elements of a cycle $C$, then $E=e_{1}+\ldots+e_{k}$ is invariant under $A^{-1}$.

Proposition S-3 (pp. 62-63). For $E$ as above there is another orthogonal decomposition $E=E_{1}+\cdots+E_{k}$ into oriented 2-planes, each invariant under $A^{-1}$ and under $\operatorname{Ad}\left(T_{A}\right)$ and such that the action of $A^{-1}$ on each $E_{j}$ is rotation through $2 \pi(u+(j-1) / k)$. Here $0 \leqq u<1 / k$ and $u$ depends only on $E$. If $Y \in \mathrm{t}_{A}$ and $y=\exp (Y)$, then $\operatorname{Ad}(y)$ rotates each $E_{j}$ through $\pm 2 \pi \theta(Y)$, the choice of sign being the samefor all $j$, where $\theta$ is the common restriction of the elements of $C$ to $\mathrm{t}_{\boldsymbol{A}}$.

These propositions lead us to define functionals $w_{i j}$ on $t_{A}$ as follows. For each $E$ as in the above proposition choose $\phi$ to be the linear functional on $t_{A}$ such that $\operatorname{Ad}(y)$ rotates each $E_{j}$ through $2 \pi \phi(Y)$ ( $y$ and $Y$ as above). Let $\phi_{1}, \cdots, \phi_{h}$ be the resulting set of functionals. Let $k_{i}$ be the number of roots which restrict to $\phi_{i}$. Let $u_{i}$ be the number $u$ of Proposition S-3 for the $E$ corresponding to $\phi_{i}$. Then define 


$$
w_{i j}=\phi_{i}+u_{i}+(j-1) / k_{i}, \quad i=1, \cdots, h ; j=1, \cdots, k_{i} .
$$

Definition. The singular planes of $t_{A}$ are the hyperplanes $w_{i j} \equiv 0(\bmod 1)$. Each plane is given multiplicity equal to the number of forms $w_{i j}$ which are identically integral on it.

The following proposition should now be fairly evident:

Proposition 1.1. If $Y \in \mathrm{t}_{A}$ is contained in exactly $r$ singular planes (counted with multiplicity), if $y=\exp (Y)$, and if $G_{y}=\left\{x \in G: A(x) y x^{-1}=y\right\}$, then $\operatorname{dim}\left(G_{y}\right)=\operatorname{dim}\left(\mathrm{t}_{\boldsymbol{A}}\right)+2 r$.

This, then, provides a complete description of the dimensions of orbits under the $A$-twisted actions. Before extending this result to the general case we will state one more proposition which will be useful in what follows. The proof is practically immediate from the propositions of Siebenthal quoted above.

Proposition 1.2. Those functionals $w_{i j}$ for which $w_{i j}(0)=0$ are the roots of $\mathfrak{t}_{A}$ relative to $\mathrm{t}_{A}$.

2. The general case. Let $J$ and $L$ be involutive automorphisms of $G$ with fixed point groups $H$ and $K$ respectively. The corresponding Lie subalgebras are $\mathfrak{h}$ and $\mathfrak{f}$ and we have orthogonal decompositions

$$
\begin{aligned}
& \mathfrak{g}=\mathfrak{h} \oplus \mathfrak{m}, \\
& \mathfrak{g}=\mathfrak{f} \oplus \mathfrak{p} .
\end{aligned}
$$

Set $A=J L$.

Proposition 1.3. $\mathfrak{f}_{A}=\mathfrak{l} \cap \mathfrak{h} \oplus \mathfrak{p} \cap \mathfrak{m}$.

Proof. Clearly $\mathfrak{f}_{A}$ contains this algebra. Now $L\left(\mathfrak{\mathfrak { F }}_{A}\right)=\mathfrak{\mathfrak { F }}_{A}$, since if $X \in \mathfrak{f}_{A}$ then $J L(X)=X$ and so $L J L(X)=L(X)$. This gives $A^{-1} L(X)=L(X)$. Similarly $J\left(\mathfrak{f}_{A}\right)=\mathfrak{f}_{A}$. The formula $J L(X)=X$ also implies $J(X)=L(X)$, so that restrictions of these two involutions to $\mathfrak{f}_{A}$ agree. We denote by $\sigma$ this involution of $\mathfrak{f}_{A}$. The +1 eigenspace of $\sigma$ is clearly contained in $\mathfrak{f} \cap \mathfrak{h}$ and the -1 eigenspace in $\mathfrak{p} \cap \mathfrak{m}$. Therefore $\mathfrak{f}_{A}$ is contained in the direct sum of these two spaces and so equality holds. Q.E.D.

The symmetric space obtained by dividing $\left(K_{A}\right)_{0}$ (the identity component of the fixed point group of $A$ ) by the fixed point group of $\sigma$ is diffeomorphically imbedded in $\left(K_{A}\right)_{0}$ as $\exp (\mathfrak{p} \cap \mathrm{m})$, as is well known (cf. [4, pp. 329-330], for instance). Thus taking a maximal abelian subalgebra $t$ of $\mathfrak{p} \cap \mathrm{m}$ we obtain a maximal torus $T \subset \exp (\mathfrak{p} \cap \mathfrak{m})$ and this is a geodesically imbedded torus (not in general maximal) in $\exp (\mathfrak{m}) \approx G / H$. Now $t$ may be extended to a maximal abelian subalgebra $\mathfrak{t}_{A}$ of $\mathfrak{f}_{A}$ by adding on a suitable abelian subalgebra of $\mathfrak{f} \cap \mathfrak{h}$. On $\mathfrak{t}_{A}$ we have the system (1.1) of affine functionals $w_{i j}$. Consider the nonconstant restric- 
tions of these functionals to $t$. Identify any two functionals if they give rise to the same family of integral hyperplanes in $\mathfrak{t}$ (thus $\phi$ and $\theta$ are identified if and only if $\phi=n \pm \theta$ for some integer $n$ ). The resulting functionals are called the roots of the triad $(G ; K, H)$. Each root $\phi$ is assigned multiplicity $m(\phi)$ equal to the numberof $w_{i j}$ 's which restrict to $\phi$. The integral planes $\phi \equiv 0(\bmod 1)$ are called the singular planes. A singular plane $p$ is given multiplicity $m(p)$ equal to the sum of the multiplicities of roots $\phi$ for which $\phi(p)$ is an integer.

Proposition 1.4. The torus $T \subset G / H$ described above meets all the K-orbits and meets them orthogonally. If $Y \in t$ lies on no singular plane, then the tangent space to $T$ at $y=\exp (Y)$ is the orthogonal complement of the tangent space to the K-orbit of $y$ at $y\left({ }^{3}\right)$.

Before proving this proposition we must describe certain isometries of $G / H$. The imbedding

$$
G / H \approx \exp (\mathrm{m}) \subset G
$$

can be explicitly described via the map $\eta: G \rightarrow G$ defined by $\eta(x)=J(x) x^{-1}$. This map is constant along the left cosets of $H$ and defines an imbedding

$$
\eta_{*}: G / H \rightarrow G
$$

whose image is $\exp (\mathrm{m})$ (cf. $[4$, p. 330]). That is to say, the orbit of the identity under the $J$-twisted action of $G$ on itself is just $\exp (\mathfrak{m})$. The map $\eta_{*}$ carries the standard action of $\boldsymbol{G}$ on $\boldsymbol{G} / H$ over to the $J$-twisted action of $G$ on $\exp (\mathfrak{m})$. In particular, the action of $K$ in which we are interested takes this form. Finally, given $y \in \exp (m)$ we can map $\exp (\mathfrak{m})$ isometrically onto itself so as to map $y$ to $e$ (the identity of $G$ ) as follows. Since $y=s(1)$ for a suitable geodesic $s$ on $\exp (\mathrm{m})$ through $e$, we can choose $y^{1 / 2}=s\left(\frac{1}{2}\right) \in \exp (\mathrm{m})$. Then the map

$$
x \rightarrow y^{-1 / 2} x y^{-1 / 2}=J\left(y^{1 / 2}\right) x y^{-1 / 2}
$$

is as desired. Relative to this transformation of $\exp (\mathrm{m})$ the action of $K$ is carried over to the action (still $J$-twisted) of $y^{1 / 2} K y^{-1 / 2}$. This latter group is the fixed point group of the involution $\operatorname{Ad}\left(y^{1 / 2}\right)(L) \operatorname{Ad}\left(y^{-1 / 2}\right)=L_{y}$.

Now if in (1.2) we choose $y \in T$, then this transformation restricted to $T$ is just group translation by $y^{-1}$ (choosing $y^{1 / 2} \in T$, of course). $L$ is replaced by $L_{y}, J$ remains the same, and $A=J L$ is replaced by

$$
A_{y}=J L_{y}=\operatorname{Ad}\left(y^{-1}\right) \cdot A
$$

since $y \in \exp (p \cap m)$. The reader will easily check (making use of (1.3)) that if

(3) I am grateful to the referee for pointing out to me that the construction of $T$ and a proof that $T$ meets all the $K$-orbits and is orthogonal to them has already been given by Hermann in [8]. He also pointed out the general relevance of [9] to this section. 
$Y \in \operatorname{tand} y=\exp (Y)$, then the new diagram of singular planes in $t$ is the translate by $-Y$ of the old diagram.

Now we prove Proposition 1.4. If $X \in \mathfrak{F}, Y \in \mathfrak{p} \cap \mathfrak{m}$, then we have

$$
\langle J X-X, Y\rangle=-2\langle X, Y\rangle=0 .
$$

Conversely, if $Y \in \mathfrak{m}, X \in \mathfrak{f}$ and if $0=\langle J X-X, Y\rangle$, then $-2\langle X, Y\rangle=0$ and so $Y \in \mathfrak{p} \cap \mathfrak{m}$. This proves that $\mathfrak{p} \cap \mathfrak{m}$ is the normal to the $K$-orbit of $e$ at $e$ and it follows by Morse theory that every $K$-orbit in $\exp (\mathfrak{m})$ meets $\exp (\mathfrak{p} \cap \mathfrak{m})$. Now for any such orbit let $b$ be a point of the orbit in $\exp (\mathfrak{p} \cap \mathfrak{m}) . b$ lies on some maximal torus of $\exp (\mathfrak{p} \cap \mathfrak{m})$ and so by the conjugacy theorem for maximal tori in a symmetric space (cf. [6, p. 1020]) there is $x \in K \cap H$ such that $J(x) b x^{-1}=x b x^{-1} \in T$. This proves that every orbit meets $T$. It also proves that the orbit of $e$ meets $T$ orthogonally at $e$. Now using a basic transformation (1.2) we see that the orbit of any $y \in T$ meets $T$ orthogonally at $y$. There remains only the last assertion of the proposition. We may assume $Y=0, y=e$. Then the assumption on $Y$ implies (by Proposition 1.2) that the roots of $\mathfrak{f}_{A}$ relative to $t_{A}$ all vanish identically on $t$. The symmetric space $\exp (\mathfrak{p} \cap \mathfrak{m})$ must therefore reduce to its maximal torus, so $\mathfrak{p} \cap \mathfrak{m}=\mathrm{t}$. Since $\mathfrak{p} \cap \mathfrak{m}$ is the normal to the $K$-orbit of $e$ at $e$, the proof is complete.

It is now easy to prove Theorem 1.1. Again we can suppose $Y=0, y=e$. Let $K_{e}$ be the subgroup of $K$ which stabilizes $e$. Then $K_{e} \subset H$ and so $K_{e}$ equals $K \cap H$. It follows that $\mathfrak{f}_{e}=\mathfrak{f} \cap \mathfrak{h}$. In particular, if no singular plane of the diagram contains 0 , then by Proposition $1.2 \mathfrak{f}_{T}=\mathfrak{f} \cap \mathfrak{h}$ and the orbit of $e$ has dimension $=\operatorname{dim}(K)$ $-\operatorname{dim}\left(K_{T}\right)$. By Proposition 1.4 this is an orbit of maximal dimension. Thus, in general, the defect $d(e)$ of the $K$-orbit of $e$ is $\operatorname{dim}\left(K_{n}\right)-\operatorname{dim}\left(K_{T}\right)$. Since always $K_{T} \subset H$, we see that this number is also the defect of the orbit of $e$ in $\exp (\mathfrak{p} \cap \mathfrak{m})$ under the adjoint action of $\exp (\mathfrak{f} \cap \mathfrak{h})$. But this latter defect is the sum of the multiplicities of the singular planes of the diagram for the symmetric space $\exp (\mathfrak{p} \cap \mathfrak{m})$ which contain 0. By Proposition 1.2 these singular planes are also the singular planes of the diagram for the triad $(G ; K, H)$ which contain 0 and their multiplicities are the same in both diagrams. This proves the theorem.

We add one more definition concerning the root diagram of $(G ; K, H)$ which will be useful in §II.

Definition. Let $\theta$ be a root of $(G ; K, H)$ relative to t. Let $\bar{\theta}$ be the linear part of $\theta$. Then the basic translation $h_{\theta}$ is defined as the vector in $t$ which is orthogonal to the null-plane of $\bar{\theta}$ and satisfies $\bar{\theta}\left(h_{\theta}\right)=2$.

It should be noted that by a suitable transformation (1.2) it may be supposed that $\theta=\bar{\theta}$. Then $h_{\theta}$ becomes a basic translation for the root diagram in $t$ of the symmetric space $\exp (\mathfrak{p} \cap \mathfrak{m})\left(\mathrm{cf} .\left[4\right.\right.$, p. 331]). Then it is well known that $\exp \left(h_{\theta}\right)=e$. Extending $\mathrm{t}_{A}$ to a Cartan subalgebra $\mathrm{t}^{\prime}$ of $\mathrm{g}$ we see that $h_{\theta}$ must lie in the central lattice of $t^{\prime}$. Thus $\psi\left(h_{\theta}\right)$ is an integer for every root $\psi$ of $g$ relative to $t^{\prime}$. Now for any root $\phi$ of $(G ; K, H), \Phi$ is the restriction to $t$ of a root of $g$ in $t^{\prime}$. Thus: 
Proposition 1.5. If $\phi, \theta$ are roots of $(G ; K, H)$, then $\phi\left(h_{\theta}\right)$ is an integer. The integers obtained in this way are called the Cartan integers of $(G ; K, H)$.

3. An example: the Cayley plane. The compact exceptional group $F_{4}$ admits $H=\operatorname{Spin}(9)$ and $K=(\mathrm{Sp}(3) \times \mathrm{SU}(2)) / \mathbf{Z}_{2}$ as symmetric subgroups. $W=F_{4} / \operatorname{Spin}(9)$ is the Cayley projective plane, a symmetric space of rank one. Consequently, the torus $T \subset W$ which serves as fundamental domain for the action of $K$ must be of dimension one. The root diagram for the triad can be shown to consist of the four functionals $x, x+\frac{1}{2}, 2 x, 2 x+\frac{1}{2}$ (where $x$ is a real variable) with respective multiplicities $4,4,3,4$. The singular subtori of $T=S^{1}$ are four points equally spaced around the circle and with respective multiplicities $7,4,7,4$. The two singular points of defect 7 lie on a common $K$-orbit as do the two of defect 4 . Let $N_{1}$ be the singular orbit of defect 4 and let $N_{2}$ be the one with defect 7 . Set $\Omega_{i}$ equal to $\Omega\left(W ; y, N_{i}\right)$ where $y$ is a point of $T$ of zero defect.

The above information together with standard Morse theory techniques shows that $\Omega_{1}$ is obtained, up to homotopy type, by attaching a 7-cell to a point, then an 11-cell to this, and finally higher cells to this. Thus

$$
\pi_{i+1}\left(W, N_{1}\right)=\pi_{i}\left(\Omega_{1}\right)=\pi_{i}\left(S^{7}\right), \quad i<10 .
$$

From this together with the homotopy exact sequence of a pair we obtain

$$
\pi\left(N_{1}\right)=\pi_{i}(W)=0, \quad i<7,
$$

the second equality being well known for the Cayley plane. Now $\operatorname{dim}(W)=16$, so $\operatorname{dim}\left(N_{1}\right)=11$. The acyclicity of $N_{1}$ in dimensions less than 7 together with Poincaré duality shows that $N_{1}$ has the homology groups of the sphere $S^{11}$. By Smale's proof of the generalized Poincaré conjecture [13], it follows that $N_{1}=S^{11}$.

Similar reasonings on the other singular orbit $N_{2}$ reveal that $N_{2}$ is an eight dimensional manifold with homology groups

$$
H_{i}\left(N_{2}\right)= \begin{cases}\mathbf{Z}, & i=0,4,8 \\ 0, & \text { otherwise }\end{cases}
$$

Presumably $N_{2}$ is the quaternion projective plane.

II. The Bott-Samelson $K$-cycles. We retain the notations of the previous section with a few additions. If $p$ is a singular plane in $t$ we denote by $K_{p}$ the subgroup of $K$ whose action leaves $\exp (p)$ pointwise fixed. Following Bott and Samelson [6] we define for each finite sequence $P=\left(p_{1}, \cdots, p_{r}\right)$ of singular planes the manifold

$$
\Gamma_{P}=K_{1} \times_{K_{T}} K_{2} \times_{K_{T}} \cdots \times_{K_{T}}\left(K_{r} / K_{T}\right)
$$

where for brevity we have written $K_{i}=K_{p_{i}}$. Such a manifold we call a $K$-cycle. For $\Omega$ as in the introduction and $s \in \Omega$ a geodesic segment transversal to the 
$K$-orbits, the $K$-cycle $\Gamma_{s}$ as defined in [6, p. 970], can be assumed to be of the form $\Gamma_{P}$ without any loss of generality. It is our intention in this section to examine carefully the geometric and topological structure of the $K$-cycles $\Gamma_{p}$. The motivation for this discussion has already been explained in the introduction.

Our geometric result is Theorem 2.1 and our main topological result is Theorem 2.2. The statement of Theorem 2.2 contains the term "regular" which we are finally prepared to define.

Definition. The symmetric triad $(G ; K, H)$ is said to be regular if and only if for every pair $\phi, \theta$ of roots of the triad with mutliplicities $m(\phi)$ odd and $m(\theta)=1$, the Cartan integer $\bar{\phi}\left(h_{\theta}\right)$ is even.

1. The manifold $K_{p} / K_{T}$. In carrying out the computation of $K_{p} / K_{T}$ we may assume (via a transformation (1.2)) that $0 \in p$. One consequence of this assumption is that the functionals (1.1) on $t_{A}$ which are identically integral on $p$ are actually linear and so are roots of $\mathfrak{f}_{A}$ relative to $t_{A}$ (cf. Proposition 1.2). Let $\phi_{1}, \cdots, \phi_{r}$ be the distinct ones that vanish on $p$ but not on all of $t$, and let $R$ be the set of roots of $\mathfrak{f}_{A}$ relative to $t_{A}$ which are integral linear combinations of the roots $\phi_{i}$. Identify $R$ with a subset of $\mathrm{t}_{A}$ via the invariant inner product. Finally set

$$
\gamma_{p}=\operatorname{span}\left\{R, e_{\alpha}: \alpha \in R\right\}
$$

where $e_{\alpha}$ is the 2-plane in $\mathfrak{f}_{A}$ orthogonal to $\mathrm{t}_{A}$ and invariant under $\operatorname{ad}\left(\mathrm{t}_{A}\right)$. The following lemma is proven easily from general Lie theory:

LemMa 2.1. $\gamma_{p}$ is a semi-simple Lie subalgebra of $\mathfrak{f}_{A}$. In fact, $\gamma_{p}$ is a compact form. The involution $\sigma$ defined by $L$ and $J$ in $\mathfrak{F}_{A}$ restricts to an involution $\tau$ of $\gamma_{p}$. Thus there is an orthogonal decomposition $\gamma_{p}=\mathfrak{f}_{\tau} \oplus \mathfrak{m}_{\mathfrak{\tau}}$ where $\mathfrak{f}_{\tau}$ is the fixed point algebra of $\tau$ and $m_{\tau}$ the -1 eigenspace.

LemMa 2.2. $\exp \left(\mathfrak{m}_{\mathfrak{\tau}}\right)$ is a symmetric space of rank one.

Proof. Evidently $\left[\gamma_{p}, p\right]=0$. Thus if $l$ is maximal abelian in $\mathfrak{m}_{\mathfrak{r}}, p \oplus l$ is abelian in $\mathfrak{p} \cap \mathfrak{m}$. Since $t$ is maximal abelian in $\mathfrak{p} \cap \mathfrak{m}$ it follows that $\operatorname{dim}(l)=1$. Q.E.D.

Now set $K_{\tau}=\exp \left(\mathfrak{f}_{\mathfrak{r}}\right)$.

Lemma 2.3. $K_{\tau} / K_{\tau} \cap K_{T}=S^{m(p)}$.

Proof. By Lemma 2.2 and the conjugacy of maximal tori in a symmetric space the adjoint action of $K_{\tau}$ on $\mathfrak{m}_{\tau}$ is transitive on the one-dimensional subspaces. Furthermore it is well known that given $X \in \mathfrak{m}_{\mathfrak{\tau}}$ there is $y \in K_{\mathfrak{\tau}}$ such that $\operatorname{Ad}(y) X$ $=-X$ (the Weyl group is generated by the reflections in the singular planes which pass through 0$)$. Thus $\operatorname{Ad}\left(K_{\imath}\right)$ is transitive on the unit sphere in $\mathfrak{m}_{\mathfrak{r}}$. Now choosing $X \in \mathfrak{m}_{\mathfrak{r}} \cap$ t of unit length, we see that the stabilizer of $X$ in $K_{\mathfrak{r}}$ is $K_{\mathfrak{r}} \cap K_{T}$. The dimension of the quotient $K_{\tau} / K_{\tau} \cap K_{T}$ has to be the multiplicity of $\{0\}$ as a singular plane in span $\{X\}$. This number is $m(p)$ since $R$ is identified with the roots of $\gamma_{p}$ and the elements of $R$ which fail to vanish on $\operatorname{span}\{X\}$ are $\phi_{1}, \cdots, \phi_{r}$. Q.E.D. 
Now by dimensionality considerations and the Brouwer theorem of invariance of domain it follows immediately that the inclusion $K_{\tau} \subset\left(K_{p}\right)_{0}$ induces a homeomorphism

$$
\left(K_{p}\right)_{0} /\left(K_{p}\right)_{0} \cap K_{T} \approx S^{m(p)} .
$$

This result would be adequate for our ultimate purposes since only the principal component of $\Gamma_{P}$ affects the topology of $\Omega$, but the following proposition is more satisfying and does simplify matters somewhat $\left({ }^{4}\right)$.

Proposition 2.1. $K_{T}$ intersects every component of $K_{p}$, hence $K_{p} / K_{T} \approx S^{m(\rho)}$.

Proof. Let $y \in K_{p}$. The proposition will be proven by finding $u \in\left(K_{p}\right)_{0}$ such that $u y \in K_{T}$. Since $0 \in p$ we have $K_{p} \subset H$ and so $\operatorname{Ad}(y) \mathrm{t}=\mathrm{t}^{\prime}$ is also a maximal abelian subalgebra of $\mathfrak{p} \cap \mathfrak{m}$ and centralizes $p$. The subgroup $\left(K_{A}\right)_{p}$ of $\left(K_{A}\right)_{0}$ which centralizes $p$ is evidently invariant under the involution $\sigma$ and so contains a symmetric space in which both $T$ and $T^{\prime}=\exp \left(\mathrm{t}^{\prime}\right)$ are maximal tori. The identity component of the fixed point group of $\sigma$ in $\left(K_{A}\right)_{p}$ is $\left(K_{p}\right)_{0}$, and so we can find $x \in\left(K_{p}\right)_{0}$ such that $\operatorname{Ad}(x) t^{\prime}=t$. Finally, we recall that for $X \in \mathfrak{m}_{\tau} \cap t$ as in the proof of Lemma 2.3 there is $z \in K_{\tau} \subset\left(K_{p}\right)_{0}$ such that $\operatorname{Ad}(z) X=-X$. Thus either $x$ or $z x$ will be the desired element $u \in\left(K_{p}\right)_{0}$. Q.E.D.

Return for a moment to the example involving the Cayley plane $(\S I, 3)$. The nonsingular $K$-orbits are all diffeomorphic to $K / K_{T}$. We can now see that this is a fiber bundle over $K / K_{p}=N_{1}=S^{11}$ (where $m(p)=4$ ) with fiber $K_{p} / K_{T}=S^{4}$.

2. The structure of $\Gamma_{P}$. It is now fairly easy to prove Theorem 2.1. For the proof that $\Gamma_{P}$ is an iterated fiber bundle over $K_{1} / K_{T}$ with fibers $K_{i} / K_{T}$ and with canonical global cross-sections $s_{i}$ the reader is referred to [6, p. 997]) By Proposition 2.1 these fibers are the spheres $S^{m\left(p_{t}\right)}$.

Now for a singular plane $p$ through the origin, Proposition 2.1 gives a canonical map

$$
\pi: K_{p} \rightarrow \mathfrak{m}_{\tau}
$$

whose image is the unit sphere in $m_{\tau}$. It is easy to see that $\pi$ induces an isomorphism $\pi_{*}$ of $V$, the orthogonal complement of $\mathfrak{f}_{T}$ in $\mathfrak{f}_{p}$, onto the tangent plane to this sphere at $X \in \mathfrak{m}_{\tau} \cap t$. The translation $\lambda$ in $\mathfrak{m}_{\tau}$ defined by

$$
\lambda(Y)=Y-X
$$

takes this tangent plane to the orthogonal complement of $\operatorname{span}\{X\}$ in $\mathfrak{m}_{\tau}$. For $x \in K_{T}$ it is easy to see that

(4) In my thesis I defined the $K$-cycle corresponding to $P$ to be the principal component of $\Gamma_{P}$ and obtained Theorem 2.1 for that object. From Proposition 2.1, however, it is clear that $\Gamma_{P}$ is always connected. I am indebted to Professor Araki for communicating to me a proof of this fact for the case $K=H$. His proof inspired the proof given here for the general case. 


$$
\lambda \pi_{*}(\operatorname{Ad}(x) Z)=\operatorname{Ad}(x) \lambda \pi_{*}(Z)
$$

for all $Z \in V$. Finally note that $\operatorname{Ad}\left(K_{T}\right)$ leaves $X$ fixed. These observations make it clear that if $0 \in p_{i}$, then for $E_{i}$ as in the statement of Theorem 2.1 there is a vector bundle isomorphism

$$
E_{i}+1 \approx K_{1} \times K_{T} \cdots \times_{K_{T}} K_{i-1} \times_{K_{T}}\left(\mathrm{~m}_{\tau}\right)
$$

and the sphere bundle $\Gamma_{i} \rightarrow \Gamma_{i-1}$ is the associated unit sphere bundle. The hypothesis $0 \in p_{i}$ is removed by noting that a transformation (1.2) replaces each $K_{j}$ with $\operatorname{Ad}(b) K_{j}$ and $V_{i}$ with $\operatorname{Ad}(b) V_{i}$ (where $b \in T$ ) and since $b$ commutes with everything in $K_{T}$ we obtain a homeomorphism of base spaces which lifts to an isomorphism of the vector bundles. This completes the proof of Theorem 2.1.

3. The topology of $\Gamma_{P}$. The Gysin sequence together with the cross-sections $s_{i}$ proves the following:

Proposition 2.2. If all the vector bundles $E_{i}$ are orientable, then, setting $m(i)=m\left(p_{i}\right)$,

$$
H_{*}\left(\Gamma_{P}\right) \approx H_{*}\left(S^{m(1)}\right) \otimes \cdots \otimes H_{*}\left(S^{m(r)}\right) .
$$

In particular, $\Gamma_{P}$ is an orientable manifold. In any case this formula always holds mod 2.

We shall soon develop criteria for the orientability of the $E_{i}$, but first let us briefly consider the cohomology ring of $\Gamma_{P}$. We will not completely identify this ring, but we will give an interesting partial identification. If all the $E_{i}$ are orientable, then in what follows we suppose integer coefficients; otherwise we work mod 2. By Proposition 2.2 the fibers in the successive fibrations $\pi_{i}$ are all totally nonhomologous to zero relative to the suitable coefficient ring. Thus:

Proposition 2.3. With suitable coefficients there is a class $x_{i} \in H^{m(i)}\left(\Gamma_{l}\right)$ such that

$$
H^{*}\left(\Gamma_{i}\right) \approx \pi_{i}^{*} H^{*}\left(\Gamma_{i-1}\right)+x_{i} \cup \pi_{i}^{*} H^{*}\left(\Gamma_{i-1}\right),
$$

where $\pi_{i}^{*}$ is injective.

Since the maps $\pi_{i}^{*}$ are injective we will drop reference to them in the future. It follows that $x_{i}$ can be considered as elements of $H^{*}\left(\Gamma_{P}\right)$ and that as such they generate this ring. In fact, the entire ring structure will be determined by the relations

$$
x_{i}^{2}=a_{i} x_{i}+b_{i}
$$

where $a_{i}, b_{i} \in H^{*}\left(\Gamma_{i-1}\right)$. We may choose $x_{i}$ such that $s_{i}{ }^{*}\left(x_{i}\right)=0\left(s_{i}\right.$ the canonical cross-section) and then the above relations take the form

$$
x_{i}^{2}=a x_{i}
$$


In the case in which all the $E_{i}$ are orientable, the choice of the integral classes $x_{i}$ is also a choice of orientation for each $E_{i}$. We may therefore speak of the Euler class $X\left(E_{i}\right)$ and view it as an element in $H^{*}\left(\Gamma_{P}\right)$. Likewise the top Whitney class $w_{m(i)}\left(E_{i}\right)$ will be viewed as an element in $H^{*}\left(\Gamma_{P} ; \mathbf{Z}_{2}\right)$.

PROPOSITION 2.4. If all the $E_{i}$ are orientable, then in the basic relations (2.1) $a_{i}=X\left(E_{i}\right)$. Otherwise $a_{i}=W_{m(i)}\left(E_{i}\right)$.

Proof. First assume all $E_{i}$ orientable. Consider the sequence of spaces

$$
0 \rightarrow \Gamma_{i-1} \stackrel{s_{i}}{\rightarrow} \Gamma_{i} \stackrel{\eta}{\rightarrow} \Gamma_{i} / s_{i}\left(\Gamma_{i-1}\right) \rightarrow 0
$$

where $\eta$ is the identification map. There is a natural identification

$$
T\left(E_{i}\right) \approx \Gamma_{i} / s_{i}\left(\Gamma_{i-1}\right)
$$

where $T\left(E_{i}\right)$ is the Thom space of the vector bundle $E_{i}$. Using the fact that $\pi_{i} \circ s_{i}=1$, we deduce an exact sequence

$$
0 \rightarrow \tilde{H}^{*}\left(T\left(E_{i}\right)\right) \stackrel{\eta^{*}}{\longrightarrow} H^{*}\left(\Gamma_{\imath}\right) \underset{\pi_{i}^{*}}{\stackrel{s_{i}^{*}}{\rightleftarrows}} H^{*}\left(\Gamma_{i-1}\right) \rightarrow 0,
$$

where $\tilde{H}^{*}$ stands for reduced cohomology and $\pi_{i}{ }^{*}$ splits the sequence. There is a unique class $U \in H^{m(i)}\left(T\left(E_{i}\right)\right)$ such that $\eta^{*}(U)=x_{i}$ and by standard theory the Thom isomorphism

$$
\phi: H^{q}\left(\Gamma_{i-1}\right) \rightarrow H^{q+m(i)}\left(T\left(E_{i}\right)\right)
$$

can be defined by

$$
\phi(a)=\pi_{i}^{*}(a) \cup U
$$

which makes sense via the identification

$$
\tilde{H}^{*}\left(T\left(E_{i}\right)\right) \approx H^{*}\left(\Gamma_{i}, s_{i}\left(\Gamma_{i-1}\right)\right) .
$$

By definition the Euler class is

$$
X\left(E_{i}\right)=\phi^{-1}(U \cup U)
$$

(cf. $[11$, p. 41$])$. Thus

$$
\begin{aligned}
\eta^{*} \phi\left(X\left(E_{i}\right)\right) & =x_{i}^{2}, \\
\eta^{*} \phi\left(X\left(E_{i}\right)\right) & =\eta^{*}\left(\pi_{i}^{*}\left(X\left(E_{i}\right)\right) \cup U\right) \\
& =\pi_{i}^{*}\left(X\left(E_{i}\right)\right) \cup x_{i} .
\end{aligned}
$$

This gives the desired formula. The same argument always works mod 2 and gives the second assertion. Q.E.D. 
It should be remarked that the mod 2 part of this proposition is a special case of a theorem of W. S. Massey [10, Theorem III, p. 274].

The next step in computing the cohomology ring would be an explicit determination of these characteristic classes in terms of the generators $x_{i}$, but this seems to be very complicated in the general case. For a determination (with coefficients $\mathbf{Z}_{2}$ and in some cases $\mathbf{Z}$ ) of the cohomology ring of the $K$-cycles which occur in the case $K=H$, the interested reader is referred to [2].

4. The vector bundles $E_{i}$. It is important to obtain conditions under which the $K$-cycles are all orientable. By Proposition 2.2 it will be sufficient to find conditions under which the vector bundles $E_{t}$ are all orientable.

Let $p$ be a singular plane in $t, 0 \in p$. Consider the roots of $\mathfrak{f}_{A}$ relative to $t_{A}$ which vanish on $p$ but not identically on $t$. We may orient these roots so that their restrictions to $t$ all have the same sign. It is well known (cf. [1], for instance) that there are at most two distinct restrictions $\theta, 2 \theta$ of these roots to $t$. If $\theta$ is the only restriction of these roots we write $m(2 \theta)=0$. From the table at the end of [1], it can be seen that one of the following situations always holds:

I. $m(\theta)$ even, $m(2 \theta)=0$.

II. $m(\theta)>1$ and is odd, $m(2 \theta)=0$.

III. $m(\theta)$ is even, $m(2 \theta)$ is odd.

IV. $m(\theta)=1, m(2 \theta)=0$.

Defintion. A singular plane $p$ in t is said to be of type I, II, III, or IV according to which of the above situations occurs when $p$ is moved to the origin via a transformation (1.2).

The separation of types II and IV may strike the reader as a bit arbitrary, but, as we shall see, it is only the presence of planes of type IV that can introduce nonorientability into the $K$-cycles.

Now for each possible type of $p$ we describe the system of roots of $\mathfrak{f}_{\boldsymbol{A}}$ relative to $t_{A}$ which vanish on $p(0 \in p)$ but not identically on $t$.

I. The distinct roots with restriction $\theta$ are $\theta_{1}, \cdots, \theta_{n},-\sigma\left(\theta_{1}\right), \cdots,-\sigma\left(\theta_{n}\right)$.

II. The distinct roots with restriction $\theta$ are $\phi, \theta_{1}, \cdots, \theta_{n},-\sigma\left(\theta_{1}\right), \cdots,-\sigma\left(\theta_{n}\right)$ where $\phi=-\sigma(\phi)$.

III. The distinct roots with restriction $\theta$ are $\theta_{1}, \cdots, \theta_{n},-\sigma\left(\theta_{1}\right), \cdots,-\sigma\left(\theta_{n}\right)$ and those with restriction $2 \theta$ are $\phi, \phi_{1}, \cdots, \phi_{m},-\sigma\left(\phi_{1}\right), \cdots,-\sigma\left(\phi_{m}\right)$ where $\phi=-\sigma(\phi)$.

IV. $\phi$ restricts to $\theta$ and $\phi=-\sigma(\phi)$.

These assertions are readily seen using the fact that the system of roots in question is closed under the involution $\sigma$.

LeMma 2.4. Let $\phi$ be a root of $\mathfrak{f}_{A}$ relative to $\mathrm{t}_{A}$. Let $e_{\phi}$ be the 2-plane in $\mathfrak{f}_{A}$ corresponding to $\phi$. If $\sigma(\phi)=\phi$ then $e_{\phi}$ is pointwise fixed under $\sigma$. If $\sigma(\phi)=-\phi$, then $e_{\phi}$ is spanned by $X_{\phi}, Y_{\phi}$ such that $\sigma\left(X_{\phi}\right)=-X_{\phi}, \sigma\left(Y_{\phi}\right)=Y_{\phi}$.

Proof. In both cases, $\sigma\left(e_{\phi}\right)=e_{\phi}$. If $\sigma(\phi)=\phi$ and if $e_{\phi}$ is not pointwise fixed under $\sigma$, then there is a -1 eigenvector $X \neq 0$ in $e_{\phi}$. But evidently $\phi(t)=0$ and so 
$[X, \mathrm{t}]=0$, contradicting the fact that $\mathrm{t}$ is maximal abelian in $\mathfrak{p} \cap \mathfrak{m}$. For $\sigma(\phi)=-\phi$ we may choose $X_{\phi}, Y_{\phi}$ as nonzero mutually orthogonal eigenvectors of $\sigma$ in $e_{\phi}$ with eigenvalues $\varepsilon_{1}, \varepsilon_{2}$ respectively. Necessarily $\varepsilon_{i}= \pm 1$. We may suppose $Z_{\phi} \in \mathfrak{t}$ such that $Y_{\phi}=\left[Z_{\phi}, X_{\phi}\right]$. Then

$$
\varepsilon_{2} Y_{\phi}=\sigma\left(Y_{\phi}\right)=\left[-Z_{\phi}, \varepsilon_{1} X_{\phi}\right]=-\varepsilon_{1} Y_{\phi} .
$$

Therefore $\varepsilon_{1}=-\varepsilon_{2}$ and we may suppose $\varepsilon_{1}=-1, \varepsilon_{2}=1$. Q.E.D.

Now for $\phi$ as in II, III, or IV we take $Y_{\phi}$ as in Lemma 2.4. For case I we set $Y_{\phi}=0$. For $\alpha$ any root let $X_{\alpha}, Y_{\alpha}$ be an orthonormal basis of $e_{\alpha}$. Set $X(\alpha)=X_{\alpha}+\sigma\left(X_{\alpha}\right), \quad Y(\alpha)=Y_{\alpha}+\sigma\left(Y_{\alpha}\right)$.

LEMMA 2.5. The orthogonal complement of $\mathfrak{F}_{T}$ in $\mathfrak{f}_{p}$ is

$$
V=\operatorname{span}\left\{X\left(\theta_{i}\right), Y\left(\theta_{i}\right), X\left(\phi_{j}\right), Y\left(\phi_{j}\right), Y_{\phi}: i=1, \cdots, n ; j=1, \cdots, m\right\} \text {. }
$$

Proof. This is clearly a subspace of $\mathfrak{f}_{p}$. Furthermore, using the first assertion in Lemma 2.4, we see that $\mathfrak{F}_{T}$ is spanned by the orthogonal complement of $t$ in $t_{A}$ together with the planes $e_{\alpha}$ for roots satisfying $\sigma(\alpha)=\alpha$ (which is the same as saying $\alpha(t)=0)$. The space $V$ described above is evidently orthogonal to $\mathfrak{f}_{T}$. Finally, $\operatorname{dim}\left(\mathfrak{f}_{p}\right)-\operatorname{dim}\left(\mathfrak{f}_{T}\right)=m(p)=\operatorname{dim}(V)$. Q.E.D.

Now note that without the assumption that $0 \in p$ we still have that the orthogonal complement of $\mathfrak{f}_{T}$ in $\mathfrak{f}_{p}$ is $\operatorname{Ad}(b) V$ for $V$ as above and $b \in T$. One immediately deduces (using the notation of Theorem 2.1):

COROLlaRY. Let $p_{i}^{\prime}$ be the plane through 0 obtained by moving $p_{i}$ to the origin by a standard transformation. Let $V_{i}^{\prime}$ be obtained from $p_{i}^{\prime}$ as in the above lemma. Then there is a bundle isomorphism

$$
E_{i} \approx K_{1} \times{ }_{K_{T}} \cdots \times_{K_{T}} K_{i-1} \times{ }_{K_{T}} V_{i}^{\prime}
$$

With this identification of $E_{i}$ it is a fairly easy matter to determine whether the bundle is orientable or not. As is well known, $E_{i}$ is orientable if and only if the first Whitney class $w_{1}\left(E_{i}\right)=0$. We will compute this Whitney class.

Suppose $j$ an integer $\geqq 1$ and $<i$ such that $m\left(p_{j}\right)=1$. We may suppose $0 \in p_{j}$. As in [6, p. 997], there is an injection

$$
\chi_{j}: K_{j} / K_{T}=S^{1} \rightarrow \Gamma_{i} .
$$

For $x_{j} \in H^{*}\left(\Gamma_{j} ; \mathbf{Z}_{2}\right) \subset H^{*}\left(\Gamma_{i} ; \mathbf{Z}_{2}\right)$ as in Proposition 2.3, we have $\chi_{j}^{*}\left(x_{j}\right)$ equal to the generator $y$ in $\left.H^{1}\left(S^{1} ; Z_{2}\right) \cdot \chi_{j}^{*}\left(w_{1 i}\right)\right)$ is either $y$ or 0 according as $\left(E \chi_{j}^{-1}\left(E_{i}\right)\right.$ is orientable or not. Let $\theta_{j}$ be the root of $\mathfrak{A}_{A}$ relative to $t_{A}$ which vanishes on $p_{j}$ but not on all of t and let $h_{j}$ be the vector in $t_{A}$ normal to the null-plane of $\theta_{j}$ and such that $\theta_{j}\left(h_{j}\right)=2$. Then clearly $h_{j} \in \mathrm{t}$.

LeMma 2.6. If $p_{i}$ is of type I or III, then $\chi^{*}(w(E))=0$. If $p_{i}$ is of type II 
or IV, then there is a unique root $\theta$ of the triad which is identically integral on $p_{i}$ and $\chi_{j}^{*}\left(w_{1}\left(E_{i}\right)\right)=\bar{\theta}\left(h_{j}\right) y$.

Proof. Set $p=p_{j}$ and consider the group $K_{\tau}$ as in Lemma 2.3. Since $m(p)=1$ it follows that $K_{\tau}$ is a circle group and the subgroup $K_{\tau} \cap K_{T}$ is identified as $\left\{e, \exp \left(h_{j} / 2\right)\right\}$. Since we make no assumption of simple connectivity it is possible that $\exp \left(h_{j} / 2\right)=e$. Now

$$
\chi_{j}^{-1}\left(E_{i}\right) \approx K_{j} \times{ }_{K_{T}} V_{i}^{\prime}
$$

and since the inclusion $K_{\tau} \subset K_{j}$ induces a homeomorphism

$$
K_{\tau} / K_{\tau} \cap K_{T} \approx K_{j} / K_{T}
$$

(by Lemma 2.3 and Proposition 2.1) we obtain

$$
\chi_{j}^{-1}\left(E_{i}\right) \approx K_{\tau} \times{ }_{K_{\tau} \cap K_{T}} V_{i}^{\prime} .
$$

Lemma 2.5 gives a basis for $V_{i}^{\prime}$ each member of which is mapped onto \pm itself by $\operatorname{Ad}\left(\exp \left(h_{j} / 2\right)\right)$ (by standard Lie theory). Thus $\chi_{j}^{-1}\left(E_{i}\right)$ splits into a direct sum of line bundles.

Now if $p_{i}$ is of type I, $\chi_{j}^{-1}\left(E_{i}\right)$ is a direct sum of an even number of mutually isomorphic line bundles. Thus $\chi_{j}^{-1}\left(E_{i}\right)$ is trivial and its first Whitney class must vanish.

If $p_{i}$ is of type III, we argue as above to show that $\chi_{j}^{-1}\left(E_{i}\right)$ is a direct sum of two trivial bundles and a line bundle

$$
L=K_{\tau} \times_{K_{\tau} \cap K_{T}} \operatorname{span}\left\{Y_{\phi}\right\}
$$

The restriction of $\phi$ to t is $2 \bar{\theta}$ for a $\operatorname{root} \theta$ of the triad and so $\phi\left(h_{j} / 2\right)$ is an integer. It follows that $L$ is also trivial so that again the Whitney class is zero.

If $p_{i}$ is of type II or IV it is clear that the unique root $\theta$ exists as asserted. We obtain that $\chi_{j}^{-1}\left(E_{i}\right)$ is a direct sum of an odd number of line bundles, each isomorphic to

$$
L=K_{\tau} \times{ }_{K_{\tau} \cap K_{T}} \operatorname{span}\left\{Y_{\phi}\right\} .
$$

Now $\phi\left(h_{j} / 2\right)=\bar{\theta}\left(h_{j} / 2\right)$ and so $L$ is trivial if and only if this number is an integer. Thus $\chi_{j}^{*}\left(w_{1}\left(E_{i}\right)\right)=w_{1}(L)$ is $y$ or 0 accordng as $\bar{\theta}\left(h_{j}\right)$ is odd or even. Q.E.D.

Corollary 1. If $(G ; K, H)$ is regular, then $w_{1}\left(E_{i}\right)=0$ and so $E_{i}$ is orientable.

This corollary follows from the above together with the partial determination of $H^{*}\left(\Gamma_{i} ; \mathbf{Z}_{2}\right)$ achieved through Proposition 2.3. In view of Proposition 2.2 we obtain

COROLlary 2. If $(G ; K, H)$ is regular, then all the $K$-cycles are homologically torsion free. 
This is the first assertion of Theorem 2.2. For the second assertion we use the following proposition which is interesting in its own right.

Proposition 2.5. Let $P=\left(p_{1}, \cdots, p_{r}\right)$ as usual. Then $\Gamma_{P}$ is orientable if and only if

$$
\sum_{i=2}^{r} w_{1}\left(E_{i}\right)=0
$$

Proof. Let $T_{P}$ be the tangent bundle of $\Gamma_{P}, T_{i}$ that of $\Gamma_{i}$. It is sufficient to prove

$$
w_{1}\left(T_{P}\right)=\sum_{i=2}^{r} w_{1}\left(E_{i}\right)
$$

To do this we proceed inductively. First note that since $\Gamma_{1}$ is a sphere, $w_{1}\left(T_{1}\right)=0$. For the inductive step we will prove

$$
w_{1}\left(T_{i}\right)=w_{1}\left(T_{i-1}\right)+w_{1}\left(E_{i}\right),
$$

for $i>1$. Now

$$
\Gamma_{i} \vec{\pi}_{i} \Gamma_{i-1}
$$

is the unit sphere bundle of $F_{i}=E_{i}+1$. It follows readily that $\pi_{i}^{-1}\left(F_{i}\right)$ is isomorphic to $1+T_{i}^{\prime}$ where $T_{i}^{\prime}$ is the bundle of tangents along the fibers of $\pi_{i}$. Now

$$
\pi_{i}^{-1}\left(T_{i-1}\right)+T_{i}^{\prime}=T_{i}
$$

Thus

$$
T_{i}+1=\pi_{i}^{-1}\left(T_{i-1}+F_{i}\right)
$$

and so

$$
\begin{aligned}
w_{1}\left(T_{i}\right) & =w_{1}\left(\pi_{i}^{-1}\left(T_{i-1}+F_{i}\right)\right) \\
& =\pi_{i}^{*} w_{1}\left(T_{i-1}\right)+\pi_{i}^{*}\left(w_{1}\left(E_{i}\right)\right)
\end{aligned}
$$

and following our custom of dropping $\pi_{i}^{*}$ we obtain the desired formula. Q.E.D.

Now suppose that $\phi, \theta$ is a pair of roots of $(G ; K, H)$ violating the condition of regularity. That is, $m(\phi)$ is odd, $m(\theta)=1, \bar{\phi}\left(h_{\theta}\right)$ is odd. Let $p_{2}$ be a singular plane corresponding to $\phi$. Since $\bar{\phi}\left(h_{\theta}\right)$ is odd, $\Phi \neq 2 \psi$ for any other root $\psi$. It follows that $m\left(p_{2}\right)=m(\phi)$ and $p_{2}$ is of type II or IV. Let $p_{1}$ be a singular plane corresponding to $\theta$. Suppose $m\left(p_{1}\right)>1$. Then $\left(K_{1}\right)_{0} /\left(K_{1}\right)_{0} \cap K_{T}$ is a sphere of dimension $>1$ and so, by an elementary application of the exact homotopy sequence of a fibration, one obtains

$$
\left(K_{1}\right)_{0} \cap K_{T}=\left(K_{T}\right)_{0}
$$

Suppose $0 \in p_{1}$. Then

$$
b=\exp \left(h_{\theta} / 2\right) \in \exp \operatorname{span}\left\{Y_{\psi}\right\} \subset\left(K_{1}\right)_{0}
$$


where $\psi$ restricts to $\theta=\bar{\theta}$ and $\sigma(\psi)=-\psi$. Thus $b \in\left(K_{T}\right)_{0}$. $\operatorname{Ad}(b)$ is therefore an orientation preserving transformation of $V_{2}^{\prime}$ (cf. Lemma 2.5 and corollary). But $\bar{\phi}\left(h_{\theta}\right)$ odd implies $\operatorname{Ad}(b)$ is -1 on $V_{2}^{\prime}$ and then $\operatorname{dim}\left(V_{2}^{\prime}\right)=m\left(p_{2}\right)$, an odd number, implies that $\operatorname{Ad}(b)$ reverses orientation. Thus the assumption $m\left(p_{1}\right)>1$ must be false. Now set $P^{\prime}=\left(p_{1}, p_{2}\right)$.

Proposition 2.6. $\Gamma_{P^{\prime}}$ is not orientable.

Proof. By the preceding remarks together with Lemma 2.6, $w_{1}\left(E_{2}\right)=\bar{\phi}\left(h_{\theta}\right) y \neq 0$. Thus by Proposition 2.5, $\Gamma_{P^{\prime}}$ is not orientable. Q.E.D.

This completes the proof of Theorem 2.2 and so completes our analysis of the Bott-Samelson $K$-cycles.

\section{The topology of $\Omega$.}

1. The torsion in $H^{*}(\Omega)$. Let $\Omega=\Omega(G / H ; x, N)$ be the space of paths on $\boldsymbol{G} / \boldsymbol{H}$ starting at $x$ and terminating on the $K$-orbit $N . \Omega$ can be topologized by the compact-open topology. It is a well-known fact in Morse theory that $\Omega$ as defined here is of the same weak homotopy type as the corresponding space $\Omega^{\prime}$ of piecewise regular curves parametrized proportionally to arc length with the topology as described in [6, p. 968]. Thus Theorem I of [6], though stated for $\Omega^{\prime}$, also applies to $\Omega$. In what follows we will understand by $H_{*}(\Omega)$ the singular homology of $\Omega$ with integer coefficients. The principal result of this section has already been stated in the introduction as Theorem 3.1.

We remark that since $G / H$ is connected, varying the choice of $x$ does not vary the homotopy type of $\Omega$. Choosing'x as a suitable point of $T$ lying on none of the singular subtori, we find that all $K$-transversal geodesic segments through $x$ lie on $T$, as follows immediately from Proposition 1.4. By exercising care in the choice of $x$ we may also assume that for each $K$-transversal geodesic segment $s \in \Omega$ the $K$-cycle $\Gamma_{s}$ as defined in $\left[6\right.$, p. 970], is a $K$-cycle $\Gamma_{P}$ in our sense. Thus the fact that regularity implies $H_{*}(\Omega)$ to be free of torsion follows from Theorem 2.2 together with Theorem I of [6] and Hermann's result [7] that the action of $K$ on $G / H$ is variationally complete. We are left with the task of exhibiting a nonzero torsion element in $H_{*}(\Omega)$ whenever $(G ; K, H)$ is not regular.

LEMмA 3.1. Let $(G ; K, H)$ be irregular. For $\Omega$ as above and for a suitable choice of $x$ there is a K-transversal geodesic segment $s \in \Omega$ with $\Gamma_{s}=\Gamma_{p^{\prime}}$, where $P^{\prime}=\left(p_{1}, \cdots, p_{r}\right)$ is a sequence of singular planes such that, for every pair $(\phi, \theta)$ of roots of $(G ; K, H)$ exhibiting the irregularity, $\phi$ is not identically integral on $p_{j}, j \geqq 2$, while for some such $(\phi, \theta), \phi\left(p_{1}\right)$ is an integer.

Proof. In $t$ select a point $Y$ such that $\exp (Y) \in N$. Now extend a line from $Y$ in $t$ in such a direction that it is not parallel to any plane of the diagram. A small change in direction will not spoil this property and will further assure that the line crosses singular planes singly. There will be a first point (after $Y$ ) along this line 
at which the line intersects a singular plane $p_{1}$ for which $\phi\left(p_{1}\right)$ is an integer for some pair $(\phi, \theta)$ exhibiting the irregularity. Extend the segment a little past $p_{1}$ to a point $X$ lying on no singular plane. The segment $\bar{s}$ from $X$ to $Y$ obtained by suitable reparametrizing then gives $s=\exp \circ \bar{s}$ satisfying our requirements (taking $x=\exp (X))$. Q.E.D.

Now for $P^{\prime}=\left(p_{1}, \cdots, p_{r}\right)$ as in the lemma and $(\phi, \theta)$ exhibiting the irregularity and $\phi\left(p_{1}\right)$ an integer, we can conclude as in the remarks immediately preceding Proposition 2.6 that $p_{1}$ is of type II or IV. We can also (by the same remarks) choose $p_{0}$, a plane of typeV, such that $\theta\left(p_{0}\right)$ is an integer. Set $P=\left(p_{0}, p_{1}, \cdots, p_{r}\right)$. We define an imbedding

$$
\chi: \Gamma_{s} \rightarrow \Gamma_{P}
$$

by means of maps

where

$$
\begin{gathered}
\chi^{\prime}: K_{1} \times \cdots \times K_{r} \rightarrow K_{0} \times K_{1} \times \cdots \times K_{r}, \\
\chi^{\prime \prime}:\left(K_{T}\right)^{r} \rightarrow\left(K_{T}\right)^{r+1}
\end{gathered}
$$

$$
\begin{aligned}
\chi^{\prime}\left(a_{1}, \cdots, a_{r}\right) & =\left(e, a_{1}, \cdots, a_{r}\right), \\
\chi^{\prime \prime}\left(t_{1}, \cdots, t_{r}\right) & =\left(e, t_{1}, \cdots, t_{r}\right) .
\end{aligned}
$$

In $[6$, p. 971], there is defined an imbedding

$$
f_{s}: \Gamma_{s} \rightarrow \Omega \text {. }
$$

Finally, define $u \in \Omega$ as exp $\circ \bar{u}$ where $\bar{u}$ is defined by

$$
\bar{u}(t)=\bar{s}(3(t-2 / 3)), \quad 2 / 3 \leqq t \leqq 1 .
$$

$\bar{u} \mid[1 / 3,2 / 3]=$ line segment properly crossing $p_{0}$ with $\bar{u}(2 / 3)$

$$
=\tilde{s}(0) \text { (a general point on } \mathrm{t}) \text {. }
$$

$\bar{u} \mid[0,1 / 3]=$ polygonal line with $\bar{u}(1 / 3)$ as already deter-

$$
\text { mined and with } \bar{u}(0)=\tilde{\xi}(0) \text {. }
$$

Then by the construction in $[3$, p. 40], we obtain a map

$$
f_{u}: \Gamma_{P} \rightarrow \Omega \text {. }
$$

To the reader is left the task of checking that $f_{u} \circ \chi$ is homotopic to $f_{s}$. We thus assert:

LEMMA 3.2. In homology, $f_{s *}=f_{u *} \chi_{*}$.

From the construction of $\boldsymbol{P}^{\prime}$ we have (by Lemma 2.6 together with Proposition $2.5)$ that $\Gamma_{s}$ is orientable. Thus this manifold has a fundamental integral homology class $\gamma_{s}$. 
LEMMA 3.3. $f_{s *}\left(\gamma_{s}\right) \neq 0$.

Proof. Consider the commutative diagram

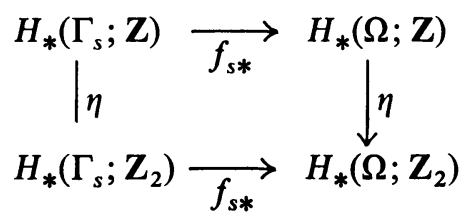

where the vertical maps $\eta$ are induced by the canonical projection $\mathbf{Z} \rightarrow \mathbf{Z}_{2}$. Now $\eta\left(\gamma_{s}\right)$ is the fundamental class mod 2 and so by Theorem I of [6], $f_{s *} \eta\left(\gamma_{s}\right)$ is an element of a vector space basis (over $\left.\mathbf{Z}_{2}\right)$ of $H_{*}\left(\Omega ; \mathbf{Z}_{2}\right)$. Thus $\eta f_{s *}\left(\gamma_{s}\right)$ $=f_{s *} \eta\left(\gamma_{s}\right) \neq 0$ and so $f_{s *}\left(\gamma_{s}\right) \neq 0$. Q.E.D.

Now

$$
\Gamma_{P}=K_{0} \times_{K_{T}} K_{1} \times_{K_{T}} \cdots \times_{K_{T}}\left(K_{r} / K_{T}\right)
$$

and so this manifold is a fiber bundle over $K_{0} / K_{T}=S^{1}$ with fiber $\Gamma_{s} \cdot \chi$ is the injection of the fiber. By Lemma 2.6 and Proposition 2.5 together with the construction of $P, \Gamma_{P}$ is not orientable. The following lemma will guarantee that $2 \chi_{*}\left(\gamma_{s}\right)=0$.

LEMMA 3.4. If $F \rightarrow X \rightarrow S^{1}$ is a locally trivial fibration with the fiber $F$ a compact oriented differentiable manifold and $X$ a nonorientable differentiable manifold, then $2 i_{*}\left(\gamma_{F}\right)=0$ where $\gamma_{F}$ is the fundamental homology class of $F$ over $\mathbf{Z}$.

Proof. If $n=\operatorname{dim}(F)$, then the action of $\pi_{1}\left(S^{1}\right)$ on $H_{n}(F)$ is nontrivial. Otherwise, from the spectral sequence of the fibration we would have to conclude that $X$ is orientable. Thus, setting $I=[0,1]$, we have a bundle map

$$
f: F \times I \rightarrow X
$$

such that the two fiber injections $f_{0}=f \mid F \times\{0\}$ and $f_{1}=f \mid F \times\{1\}$ induce $i_{*}$ and $-i_{*}$ respectively in dimension $n$. Let $\gamma_{0}$ and $\gamma_{1}$ be the respective fundamental classes of $F \times\{0\}$ and $F \times\{1\}$. Let

$$
j: F \times\{0,1\} \rightarrow F \times I
$$

be the inclusion. Consider $\gamma=\gamma_{0}-\gamma_{1} \in H_{n}(F \times\{0,1\})$. The following diagram is commutative:

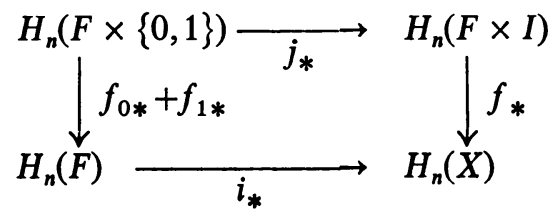

Now clearly $\left(f_{0 *}+f_{1 *}\right)(\gamma)=2 \gamma_{F}$ and so $2 i_{*}\left(\gamma_{F}\right)=f_{*} j_{*}(\gamma)=f_{*}(0)=0$. Q.E.D. 
By Lemma 3.2 and the fact that $2 \chi_{*}\left(\gamma_{s}\right)=0$ we obtain $2 f_{s *}\left(\gamma_{s}\right)=0$. By Lemma 3.3, $f_{s *}\left(\gamma_{s}\right) \neq 0$. This exhibits nonzero two-torsion in $H_{*}(\Omega)$ and so completes the proof of Theorem 3.1.

2. The special case $K=H$. The chief importance of this case is that the action of $K$ on $G / K$ has a fixed point. This means that the usual loop space of $G / K$ can be analyzed by our methods. The torus $T$ becomes a maximal torus of $G / K$ and the root system of $(G ; K, K)$ evidently reduces to the usual root system for the symmetric pair $(G, K)$. With the aid of the root diagrams which are listed in [1] it becomes a straightforward matter to check the regularity condition for each irreducible symmetric pair $(G, K)$. Calling $G / K$ regular if and only if the triad $(G ; K, K)$ is regular, we find that the regular irreducible symmetric spaces are precisely the following:

(1) Compact simple Lie groups.

(2) $G / K$ whose universal covering is one of the following spaces; complex and quaternionic Grassmann manifolds, spheres, real Grassmann manifolds of oriented 2-planes in $2 n$-space, $\mathrm{SU}(2 n) / \mathrm{Sp}(n), \mathrm{SO}(2 n) / \mathrm{U}(n), E_{6} / F_{4}$, $E_{6} /(\operatorname{Spin}(10) \cdot \operatorname{SO}(2)), E_{7} /\left(E_{6} \cdot \operatorname{SO}(2)\right), F_{4} / \operatorname{Spin}(9)$.

Thus we conclude that $G / K$ is regular if and only if each of its irreducible components is one of the above spaces.

In [2] Araki defines a $K$-cycle $\Gamma_{P}$ to be "totally orientable" if and only if each of the sphere bundles entering into its structure is orientable. He defines $(G, K)$ to be of "totally orientable type" if and only if all associated $K$-cycles are totally orientable. By Theorem 2.2 we see that this definition is equivalent to our condition of regularity. Under the assumption that $G$ is simply connected Araki establishes that the following is a sufficient condition for $(G, K)$ to be of totally orientable type: if $\mathscr{F}$ is a fundamental system of roots for $(G, K)$ then either $\mathscr{F}$ contains no root of multiplicity one, or $\mathscr{F}$ contains one root of multiplicity one and the rest of the roots in $\mathscr{F}$ have even multiplicity. By means of this criterion Araki arrives at the same list of symmetric spaces that we have given above. Thus his criterion is necessary as well as sufficient, a result which he also has obtained by an a posteriori check. We note that the assumption of simple connectivity is not essential. Theorem 2.2 involves no such assumption and so proves that $(G, K)$ is of totally orientable type if and only if its simply connected representative is. It would be interesting to have an a priori proof of the equivalence of the above condition of Araki with our regularity condition.

A check through the root diagrams of the above spaces shows many cases in which planes of multiplicity one occur; hence Theorem 3.1 applies to a number of cases in which the Morse inequalities alone would be insufficient.

3. A conjecture. Bott has conjectured that the space of loops on a compact symmetric space may have only two-torsion in homology. It is natural to extend this conjecture to all spaces $\Omega$ of the type we are here considering. A finite dimensional analogue to this conjecture would be that the spaces $K / K_{T}$, where 
$T$ is a maximal torus of $G / K$, have only two-torsion. The most that $I$ have been able to prove in all of these cases is that the torsion subgroup is generated by elements of even order. In particular, if torsion occurs in $H_{i}(\Omega)$ or in $H_{i}\left(K / K_{T}\right)$, then there is a nonzero two-primary component in that group. Furthermore, I have been able to show that the $K$-cycles $\Gamma_{P}$ have only two-torsion, so one might hope to build an infinite $K$-cycle $\Gamma$ and a map of $\Gamma$ into $\Omega$ which in cohomology would induce an injection. This would prove the conjecture for $\Omega$.

\section{REFERENCES}

1. S. Araki, On root systems and and an infinitesimal classification of irreducible symmetric spaces, J. Math. Osaka City Univ. 13 (1962), 1-34.

2. - On Bott-Samelson K-cycles associated with symmetric spaces, J. Math. Osaka City Univ. 13 (1963), 87-133.

3. R. Bott, The space of loops on a Lie group, Michigan Math. J. 5 (1958), 35-61.

4. - The stable homotopy of the classical groups, Ann. of Math. (2) 70 (1959), 313-337.

5. - An application of the Morse theory to the topology of Lie groups, Bull. Soc. Math. France 84(1956), 251-282.

6. R. Bott and H. Samelson, Applications of the theory of Morse to symmetric spaces, Amer. J. Math. 80 (1958), 964-1029.

7. R. Hermann, Variational completeness for compact symmetric spaces, Proc. Amer. Math. Soc. 11 (1960), 544-546.

8. - Totally geodesic orbits of groups of isometries, Nederl. Akad. Wetensch. Proc. Ser. A 65(1962), 291-298.

9. - On the existence of a fundamental domain for Riemannian transformation groups Proc. Amer. Math. Soc. 13 (1962), 489-494.

10. W. S. Massey, On the cohomology ring of a sphere bundle, J. Math. Mech. 7 (1958), 265-289. notes).

11. J. Milnor, Characteristic classes, Princeton Univ., Princeton, N. J., 1957 (Mimeographed

12. J. de Siebenthal, Sur les groupes de Lie compacts non connexes, Comment. Math. Helv. 31 (1956), 41-89.

13. S. Smale, The generalized Poincaré conjecture in dimensions greater than four, Ann. of Math. (2) 74(1961), 391-406.

HARVARD UNIVERSTTY,

Cambridge, Massachusetts 